\title{
An industry update: what is new in the field of therapeutic delivery this month?
}

The present industry update covers the period 1-28 February 2017. Information was sourced primarily from company press releases, regulatory and patent agencies, scientific literature and various news websites. A report issued this month indicated that the recent American election has influenced the rate and volume of pharma acquisitions and mergers (A\&M) in the final quarter of 2016 and it remains to be seen what (if any) is the effect on the therapeutic drug delivery sector in the longer term. There was positive approval news this month for Valeant, Intellipharmaceutics and Pfizer for products that offered novel therapeutic delivery. Several companies including Trutag and Novasep announced significant investment in new manufacturing facilities and strategic alliances this month.

First draft submitted: 8 March 2017; Accepted for publication: 20 March 2017; Published online: 22 May 2017

Keywords: blood-brain barrier • combination products • nanotechnology

\section{Market predictions}

Biopharma took an M\&A break last year to await US election results report A report $[1,2]$ issued by the life cycle commercial intelligence company Evaluate (London, UK) on 21 February indicated that the USA elections had a significant effect on biopharma mergers and acquisitions (M\&A) in the final quarter of 2016. The report indicated that there was a very significant decrease in M\&A activity in the fourth quarter of 2016 when just 36 deals for a total value of US $\$ 5.4$ billion in the biopharma were announced. This figure is much lower than corresponding figures of 49 deals worth $\$ 26.7$ billion for the third quarter of 2016 and 65 deals worth $\$ 29.5$ billion during the final quarter of 2015 .

\section{Drug delivery markets: forecasts to 2021}

February 2017 saw the release by the Transparency Market Research Company (NY, USA) of a report forecasting market growth to 2021 for the pulmonary and respiratory drug delivery sector [3]. This report predicts that the market will reach $\$ 52.37$ billion by 2021 from $\$ 36.10$ billion in 2016 , at a compound annual growth rate (CAGR) of $6.5 \%$ during the forecast period. The reports conclude that the pulmonary delivery will become an increasingly more popular alternate route of drug delivery and identify increasing incidence of respiratory diseases such as chronic obstructive pulmonary disease, asthma and cystic fibrosis as key market drivers. It predicts that the development of smart/digital inhalers will also be very significant during this time period.

This month also saw the release of a report forecasting the growth in the injectable drug market [4]. The New York-based Persistence Market Research Pvt's report entitled 'Injectable Drug Delivery Market: Global Industry Analysis and Forecast to 2015 to 2021' states that the drivers for the global market will be an aging population and an increase in the incidence of diabetes. The report further claims that the increase in biosimilar products will further increase the markets in this sector.
Elaine Harris

Innovation21, Unit B Deansgrange Business Park, Blackrock, Co Dublin, Ireland

Tel: +3530872988189

Elaine@innovation21.ie 
Business development

Novasep opens $€ 11 \mathrm{M}$ new antibody-drug conjugate bioconjugation unit

Novasep (Lyons, France), a contract service provider in the area of antibody-drug conjugate (ADC) production announced both a new Bioconjugation unit and viral vector production facility this month $[5,6]$. Novasep's new Bioconjugation unit is located at Le Mans, France and is estimated to have cost 11 million. The facility features two flexible GMP production suites equipped with 10-400 1 vessels and is capable of supporting both clinical and commercial manufacturing of ADCs. Novasep's new viral vector production facility will be located at Seneffe, Belgium and will be fully operational by 2019 . The new facility will feature two cGMP suites equipped with a set of single-use bioreactors ranging from 200 to $2000 \mathrm{l}$ and will have production capabilities for Phase III and commercial stages.

\section{Trutag Technologies \& Spinnaker Biosciences} form a strategic partnership

Trutag Technologies (HI, USA) announced on 23 February that they had entered a strategic partnership with Spinnaker Bioscience (CA, USA). The partnership will result in Trutag supplying its proprietary precision fabricated nanoporous silica particles to Spinnaker and will complement Spinnaker's existing drug delivery technology capabilities which were licensed from the University of California, San Diego. The lead application of the proposed output of this partnership is a novel therapeutic that is in the treatment of neovascular (wet) age-related macular degeneration [7].

\section{Assembly Biosciences license its microbiome gastrointestinal development programs to Allergan}

Assembly Biosciences (IN, USA) announced on 21 February that it had and terminated the waiting period early and had received an undisclosed upfront payment from Allergan plc (Dublin, Ireland) for a research, development, collaboration and license agreement previously announced in January 2017. This agreement will grant Allergan worldwide rights to Assembly's preclinical compounds from their microbiome gastrointestinal development programs. This includes compounds such as ABI-M201 and ABI-M301, which target irritable bowel syndromes, ulcerative colitis and Crohn's disease. Assembly Biosciences has focused on the clinical development of novel orally active therapeutics for the treatment disorders associated with the microbiome as well as hepatitis B. Assembly Biosciences' proprietary patent-pending delivery system,
GEMICEL $^{\circledR}$ is capable of targeting oral delivery of conventional therapeutics and live biologic actives to the lower GI tract [8].

\section{Developments \& clinical trials}

Antares Pharma announces US FDA acceptance of new drug application for Quickshot ${ }^{\circledR}$

\section{testosterone}

New Jersey pharmaceutical company, Antares, announced on 27 February that the FDA had accepted a new drug application for its Quickshot ${ }^{\circledR}$ testosterone product. This combination drug delivery product allows for the delivery of testosterone enanthate using a subcutaneous autoinjector and it is being proposed for the treatment of adult males diagnosed with hypogonadism. Robert F Apple, President and Chief Executive Officer of Antares commented "In addition to virtually eliminating the risk of transference that exists with topical gel products and the uncomfortable deep intramuscular administration associated with current injectable therapies, we believe that the phase three studies demonstrated that weekly subcutaneous administration of testosterone using the QuickShot autoinjector can provide patients with physiologically normal and steady levels of testosterone over the course of therapy. The study data also showed that patients had a virtually painless treatment experience using the device." A date of 20 October has been assigned by the FDA as a Prescription Drug User Fee Act date for completion of the review [9].

\section{OncoSec granted FDA fast track designation for ImmunoPulse ${ }^{\circledR}$ interleukin-12}

OncoSec (CA, USA), announced on 27 February that it had received notification of Fast Track designation from FDA for ImmunoPulse ${ }^{\circledR}$ interleukin-12 (IL-12). ImmunoPulse IL-12 is a potentially first-in-class, Intratumoral anticancer gene therapy that expresses (IL-12) for the treatment of metastatic melanoma, following progression on pembrolizumab or nivolumab. The delivery of gene therapy is facilitated through OncoSec's proprietary Oncosec Medical System. This is an electroporation system that uses an electric current, to facilitate and enhance the delivery of therapeutics into the body. Following the injection of the DNA IL-12 proteins, the Oncosec Medical System is administered. This enhances the delivery of the protein into the patients' cells. According to the company, this offers less invasive treatment which could result in a lower number of side effects to patients. The company claims that this could offer patients other treatment options compared with the conventional ones of chemotherapy and radiation therapy [10]. 
Merck receives positive Committee for

Medicinal Products for Human Use opinion for

new Pergoveris ${ }^{\circledR}$ fertility pen

On 24 February, Merck (Darmstadt, Germany)

announced that it had received a positive European Committee for Medicinal Products for Human Use opinion for its novel Pergoveris ${ }^{\circledR}$ Fertility Pen. This new product comprises of a ready-to-use liquid version of the existing Pergoveris pen and is a combination product containing both follicle-stimulating hormone (FSH) and luteinizing hormone (LH) into a single formulation for the treatment of patients with severe FSH and LH deficiency. This new product will be the only premixed combination of recombinant human FSH and $\mathrm{LH}$ on the market available in a prefilled injection device for self administration [11].

\section{Actamax submits Investigational Device Exemption to FDA for pivotal study on novel sprayable adhesion barrier device}

Pennsylvania-based Actamax Surgical Materials LLC, a wholly owned subsidiary of DSM Biomedical, announced on 21 February that it has submitted an Investigational Device Exemption to FDA to study the Actamax Adhesion Barrier in a prospective, randomized controlled trial in gynecological laparoscopic surgery. The novel sprayable adhesion barrier is a resorbable and biocompatible surgical platform that may have both delivery and antiadhesion applications [12].

\section{Early stage development \\ Tiny magnetic implant offers new drug delivery method}

University of British Columbia scientists' have developed a magnetic drug implant that they claim could offer a novel technique for controlled and programmable delivery of drugs.

The device is based on a silicone sponge containing magnetic carbonyl iron particles which are wrapped in a polymer coating. The whole device is very small, approximately $6 \mathrm{~mm}$ in diameter and the drug to be delivered is loaded into a reservoir in the implantable device. The device is then surgically implanted in the target area where the treatment is required and is subsequently activated by passing a magnet over the patient's skin at the implantation site. The activation mechanism is one whereby the sponge is compressed or deformed which then triggers the release of the drug.

According to one of the researchers Dr Chiao "This could one day be used for administering painkillers, hormones, chemotherapy drugs and other treatments for a wide range of health conditions. In the next few years we hope to be able to test it for long-term use and for viability in living models" [13].

\section{Nanodiamonds to revolutionize drug delivery?}

A fascinating report from the US Department of Energy's Oak Ridge National Laboratory, Wayne State University (TN, USA) has offered an insight into some very early stage work in the development of diamond nanoparticles approximately $5-10 \mathrm{~nm}$ in size that it is claimed can be used to enhance the delivery of RNA therapeutics. The work focuses on investigating the interactions at the interfacial layer of bionano systems. By employing a combination of experimentation and simulation, the researchers have proposed design principles for the improved delivery of RNA drugs. The research team have discovered that the transport of a tRNA (or transfer RNA) model system can be improved when conjugated to nanodiamonds and they are working on models that will allow the development of specifically designed drug-nanodiamond conjugates that could offer novel drug delivery options [14].

\section{DNA 'barcoding' allows rapid testing of nanoparticles for therapeutic delivery}

Researchers from the University of Florida, Georgia Institute of Technology (both FL, USA) and Massachusetts Institute of Technology (MIT; MA, USA) have developed a novel testing technique that involves employing tiny snippets of DNA as 'barcodes'. The researchers claim that this could lead to a novel method for rapidly screening the ability of nanoparticles in vivo to selectively deliver therapeutic genes to specific sites/ organs in a patient.

Initial experimental work carried out at the MIT labs of Professors Langer and Anderson, and demonstrated that it was possible to insert a snippet of identifiable (barcoded) DNA into each type of nanoparticle. Preclinical studies were reported studies where nanoparticles loaded with the barcoded DNA were injected into mice and the presence of the different nanoparticles were mapped for different organs in the mice. It is proposed that techniques similar to those used to sequence genomes could be employed to simultaneously test for many differently barcoded nanoparticles. In this way, it may be possible to carry out numerous preclinical investigations of different nanoparticles in a single mouse. This research was funded by the NIH and was reported in the Journal of the Proceedings of the National Academy of Sciences [15].

\section{Regulatory news \& approvals \\ Lilly's once-daily Oluminant approved by EU}

On 13 February, Lilly (IN, USA) announced that the EU had approved its once-daily product Olumiant for the treatment of Rheumatoid Arthritis (RA). Marketed 
as Olumiant, Baricitinib is the first Janus Kinase (JAK) inhibitor approved to treat RA in the EU. It is a once daily oral, selective and reversible JAK1 and JAK2 inhibitor and it is currently in the clinical studies in both the EU and US for the treatment of inflammatory and autoimmune diseases.

Currently it is estimated that there are more than 23 million people worldwide that suffer from RA. Current treatment options are principally limited to the use of NSAIDs, oral methotrexate and injectable, biological disease-modifying antirheumatic drugs; however, there is a significant unmet clinical need because many patients do not reach their therapeutic goals or sustained remission [16].

\section{Valeant receives FDA approval of SILIQTM for moderate-to-severe plaque psoriasis}

Valent Pharmaceuticals (Quebec, Canada) announced on 16 February that the FDA has approved the Biologics License Application for its monoclonal antibody that targets the IL-17 receptor for patients with moderate-to-severe plaque psoriasis product, SILIQ ${ }^{\mathrm{TM}}$ (brodalumab). SILIQTM, which is delivered as a subcutaneous injection, is indicated for the treatment of moderate-to-severe plaque psoriasis and who have failed to respond to or no longer respond to other systemic therapies. It is claimed that SILIQ ${ }^{\mathrm{TM}}$ is unique because it binds to the receptor of a protein called IL-17 and, thus, blocks the receptor from activating thereby preventing the body from receiving signals that can fuel inflammatory responses and psoriasis progression. Although successfully achieving the clinical end points, the drug is reported to have side effects including joint and muscle pain, headache, nausea, fatigue, flu, low white blood cell count, fungal infections and reactions at the injection site. The product will also carry a 'black-box' warning due to reports of people with a history of suicide attempts or depression having an increased risk of suicidal ideation and behavior [17].

\section{Lexicon Pharmaceuticals' XERMELOTM approved for the treatment of carcinoid syndrome diarrhea}

Lexicon Pharmaceuticals (TX, USA) announced on 28 February that the FDA had approved the orphan drug, XERMELO $^{\text {TM }}$ (telotristat ethyl) $250 \mathrm{mg}$. This treatment option is the first orally administered therapy approved for the treatment of carcinoid syndrome diarrhea in combination with somatostatin analog therapy in adults whose symptoms are inadequately controlled by somatostatin analog therapy alone. XERMELO ${ }^{\text {TM}}$ 's mode of action is to target tryptophan hydroxylase production within mammalian native elongating transcript cells.
Carcinoid syndrome is a group of symptoms seen in patients with carcinoid tumors. These tumors are rare and typically slow growing. They are most often found in the GI tract. Carcinoid syndrome occurs in less than $10 \%$ of patients with carcinoid tumors, usually after the tumor has spread to the liver. These tumors can release excess amounts of the hormone serotonin, which can result in diarrhea [18].

\section{AstraZeneca's Qtern approved for the treatment of Type 2 diabetes}

AstraZeneca (London, UK) announced on 28 February that the FDA has approved its once daily Qtern for the treatment of Type 2 diabetes. Qtern is a combination of $10 \mathrm{mg}$ dapagliflozin and $5 \mathrm{mg}$ saxagliptin, and is indicated as an adjunct to diet and exercise to improve glycaemic control in adults with Type 2 diabetes if they show inadequate control with dapagliflozin or for those who are already treated with dapagliflozin and saxagliptin.

The new dosage form combines $5 \mathrm{mg}$ of saxagliptin, a dipeptidyl peptidase- 4 inhibitor, with $10 \mathrm{mg}$ dapagliflozin, a sodium-glucose cotransporter 2 inhibitor. Both actives are commonly coprescribed and coadministered so their combination in a single daily dose offers a fixed-dose combination which will provide an additional oral medicine option for patients [19].

\section{Intellipharmaceutics announces FDA approval} for 500 and 750 mg generic Glucophage ${ }^{\circledR}$ XR On 28 February, the Toronto-based company, Intellipharmaceutics International Inc. announced that the FDA had approved its 500 and $750 \mathrm{mg}$ generic extended release form of Glucophage ${ }^{\circledR}$. The FDA accepted the abbreviated new drug application for 500 and $750 \mathrm{mg}$ metformin hydrochloride extended release tablets as generic equivalents to the corresponding strengths of the branded product Glucophage XR sold in the USA by Bristol-Myers Squibb. Glucophage is indicated for use in the management of Type 2 diabetes treatment.

Intellipharmaceutics have a patented Hypermatrix $^{\mathrm{TM}}$ technology, it is a multidimensional controlledrelease drug delivery platform and can be used to provide extended release formulations for actives in therapeutic areas such as neurology, cardiovascular, GI tract, diabetes and pain [20].

\section{Approval for Pfizer's novel oral RA treatment}

Pfizer Inc. (NY, USA) announced on 24 February that the approval by the FDA of its once-daily XELJANZ ${ }^{\circledR}$ XR (tofacitinib citrate) extended release 11-mg tablets for the once daily treatment of moderate-to-severe RA. Approval was received for patients who have developed intolerance to or who have failed to respond to 
methotrexate. XELJANZ XR is the first and only once daily oral RA treatment JAK inhibitor. XELJANZ XR is indicated in adults with moderately to severely active RA and may be used either in combination with methotrexate or other nonbiologic DMARDs or as a single agent.

RA affects approximately 23.7 million people worldwide and an estimated 1.5 million people in the USA. It usually occurs between 40 and 70 years of age, although it can develop at any time during adulthood [21].

\section{Patents}

Actinium announces expansion of Intellectual Property Portfolio with notice of allowance for US patent related to Actimab-A, Actimab-M and the Company's technology platform.

Actinium Pharmaceuticals Inc (NY, USA) announced on 28 February, that it had received notice of allowance from the United States Patent and Trademark Office for a patent for methods of generating a radioimmunoconjugate. This conjugated species comprises of actinium-225, an $\alpha$-emitting radioisotope and monoclonal antibodies. The allowance of this patent further strengthens actinium's $\alpha$-particle immunotherapy platform. The focus of this platform is a new form of therapeutic targeting, employing radioimmunotherapy which allows for antibodies which are conjugated to the radioisotope. The platform is based on attaching the monoclonal antibodies to the radioisotope Actinium-225. Actinium claims that their Actinium225 -conjugated therapies could result in potentially more effective and tolerable therapies [22].

\section{References}

12016 leaves pharma facing stormy waters, while the medtech sector faces an innovation imperative. www.evaluategroup.com/public/PressReleases/EP-Vantage2016-Leaves-Pharma-Facing-Stormy-Waters-While-MedtechSector-Faces-Innovation-Imperative.aspx

2 Shire and Pfizer aside, pharma took an M\&A break last year to await U.S. election results: report. www.fiercepharma.com/m-a/pharma-took-m-a-break-lastyear-to-await-u-s-election-results-report

3 Pulmonary/respiratory drug delivery market by formulation, device type, canister, end user, applications - Forecasts to 2021.

www.reportlinker.com/p04724242-summary/PulmonaryRespiratory-Drug-Delivery-Market-by-Formulation-DeviceType-Canister-End-User-Applications-Forecasts-to.html

4 Injectable drug delivery market estimated to flourish by 2021 - persistence market research. www.medgadget.com/2017/02/injectable-drug-deliverymarket-estimated-to-flourish-by-2021-persistence-marketresearch.html

5 Novasep opens $€ 11 \mathrm{M}$ new antibody-drug conjugate (ADC)

\section{Mayo seeks patent on noninvasive drug} delivery for brain cancer

The Mayo Clinic (NY, USA) announced in February that the publication of its Patent Cooperation Treaty patent application for its novel method, the so-called 'K16ApoE method', for transporting therapeutics across the blood-brain barrier. The method is of particular interest because it is claimed that the transport of the drugs occurs without modifying them using a viral vector and a synthetic peptide (K16ApoE). The inventors called it "the least complicated, least expensive and most versatile method for delivering therapeutics to the brain". It builds earlier research focused on delivering antibodies targeted against amyloid plaques into the brains of mouse models of Alzheimer's disease using the same method [23].

\section{Financial \& competing interests disclosure}

E Harris is a partner in Innovation21 Ltd, providing consultancy, advisory and evaluation services to pharmaceutical/ biotechnology companies and government funded agencies.

Apart from those already disclosed, the authors have no other relevant affiliations or financial involvement with any organization or entity with a financial interest in or financial conflict with the subject matter or materials discussed in the manuscript. This includes employment, consultancies, honoraria, stock ownership or options, expert testimony, grants or patents received or pending, or royalties.

No writing assistance was utilized in the production of this manuscript.

bioconjugation unit.

www.novasep.com/home/about-novasep/media-events/pressrelease/novasep-opens-11m-new-antibody-drug-conjugatebioconjugation-unit.html

6 Novasep invests in a $€ 27 \mathrm{~m}$ viral vector production facility. www.novasep.com/home/about-novasep/media-events/pressrelease/novasep-invests-in-a-viral-vector-production-facility. html

7 Trutag Technologies and Spinnaker Biosciences enters strategic partnership for controlled-release drug delivery. www.clinicalleader.com/doc/trutag-technologies-spinnakerbiosciences-enters-strategic-partnership-delivery-0001

8 Assembly Biosciences announces the closing and early termination of Hart-Scott-Rodino waiting period for license of microbiome gastrointestinal development programs to allergan.

http://investor.assemblybio.com/releasedetail. cfm? releaseid $=1012605$

9 Antares Pharma announces FDA acceptance of New Drug Application for Quickshot ${ }^{\circledR}$ testosterone. www.antarespharma.com/application/files/5914/8823/5269/ QST_NDA_ACCEPTANCE_Final.pdf

10 OncoSec granted FDA Fast track designation for 
ImmunoPulse ${ }^{\circledR}$ IL-12 for the treatment of metastatic melanoma following progression on pembrolizumab or nivolumab.

http://ir.oncosec.com/press-releases/detail/1896/oncosecgranted-fda-fast-track-designation-for

11 Merck receives positive CHMP opinion for new Pergoveris ${ }^{\circledR}$ fertility pen.

www.merckgroup.com/en/media/extNewsDetail.html?new sId $=$ 8D6DED31C4B31AFDC12580D000321A79\&news T ype $=1$

12 Actamax ${ }^{\mathrm{TM}}$ submits IDE to FDA for pivotal study on novel sprayable adhesion barrier device.

www.prnewswire.com/news-releases/actamax-submits-ide-

to-fda-for-pivotal-study-on-novel-sprayable-adhesion-barrierdevice-614360953.html

13 Tiny magnetic implant offers new drug delivery method. https://phys.org/news/2017-02-tiny-magnetic-implant-drugdelivery.html

14 Nanodiamonds revolutionize drug delivery. www.cemag.us/news/2017/02/nanodiamonds-revolutionizedrug-delivery

15 DNA 'barcoding' allows rapid testing of nanoparticles for therapeutic delivery.

https://phys.org/news/2017-02-dna-barcoding-rapidnanoparticles-therapeutic.html

16 European Commission approves once-daily olumiant tablets for treatment of adults with moderate-to-severe active rheumatoid arthritis.

https://investor.lilly.com/releasedetail.

cfm?ReleaseID $=1011661$

17 Valeant receives FDA approval Of SILIQ ${ }^{\mathrm{TM}}$ (brodalumab) for moderate-to-severe plaque psoriasis.

www.prnewswire.com/news-releases/valeant-receives-fdaapproval-of-siliq-brodalumab-for-moderate-to-severe-plaquepsoriasis-300408501.html

18 FDA approves Lexicon drug XERMELO ${ }^{\mathrm{TM}}$ (telotristat ethyl) $250 \mathrm{mg}$ as first and only oral treatment for carcinoid syndrome diarrhea in cancer patients with metastatic neuroendocrine tumors.

www.prnewswire.com/news-releases/fda-approves-lexicondrug-xermelo-telotristat-ethyl-250-mg-as-first-and-onlyoral-treatment-for-carcinoid-syndrome-diarrhea-in-cancerpatients-with-metastatic-neuroendocrine-tumors-300415239. html

19 FDA approves once-daily QTERN ${ }^{\circledR}$ (dapagliflozin and saxagliptin) tablets for adults with Type-2 diabetes. www.astrazeneca.com/media-centre/press-releases/2017/ fda-approves-once-daily-qtern-dapagliflozin-and-saxagliptintablets-for-adults-with-type-2-diabetes-240217.html

20 Intellipharmaceutics Announces FDA Approval for $500 \mathrm{mg}$ and $750 \mathrm{mg}$ Generic Glucophage ${ }^{\circledR}$ XR.

www.intellipharmaceutics.com/releasedetail.

cfm?ReleaseID $=1014401$

21 Pfizer announces FDA approval of XELJANZ ${ }^{\circledR}$ XR (tofacitinib citrate) extended-release tablets, the first and only once-daily oral JAK inhibitor treatment for rheumatoid arthritis.

www.pfizer.com/news/press-release/press-release-detail/ pfizer_announces_fda_approval_of_xeljanz_xr_tofacitinib_ citrate_extended_release_tablets_the_first_and_only_ once_daily_oral_jak_inhibitor_treatment_for_rheumatoid_ arthritis

22 Actinium announces expansion of intellectual property portfolio with notice of allowance for U.S. patent related to Actimab-A, Actimab-M and the Company's Technology Platform.

www.actiniumpharma.com/news-media/press-releases/ detail/221/actinium-announces-expansion-of-intellectualproperty

23 Mayo seeks patent on non-invasive drug delivery for brain cancer, Alzheimer's.

http://tcbmag.com/News/Recent-News/2017/February/ Mayo-Seeks-Patent-On-Non-Invasive-Drug-Delivery-Fo 